\title{
Ste20-like kinase is upregulated in glioma and induces glioma invasion
}

\author{
K. WANG*, R. L. HONG*, J. B. LU, D. L. WANG* \\ Department of Pathology, Medical College, Nantong University, 19 Qixiu Road, Nantong, Jiangsu 226001, P.R. China \\ *Correspondence: 18862805963@163.com \\ "Contributed equally to this work.
}

Received March 18, 2017/ Accepted May 29, 2017

\begin{abstract}
The malignant development and poor prognosis of gliomas are associated with a high degree of invasion and a high recurrence rate. However, the molecular mechanism underlying the invasiveness of glioma remains to be elucidated. Ste20like kinase (SLK) is one of the members of the Ste20 family, which has been implicated in cellular migration and invasion. In this study, we intended to explore the expression of SLK significantly related to clinicopathologic stages of gliomas. Immunohistochemical staining and western blot analysis demonstrated that SLK was highly expressed in human glioma tissues and cell lines. Kaplan-Meier analysis revealed that poor survival was associated with high SLK expression. The inhibition of SLK by RNA interference significantly suppressed the invasion ability of glioma, and on protein level, knockdown of SLK leaded to an up-regulation of E-cadherin and a down-regulation of Vimentin in glioma cells. Collectively, this research shed light on mechanisms of invasion and progression of malignant gliomas and suggested that SLK may be a potential therapeutic strategy for glioma.
\end{abstract}

Key words: glioma, SLK, E-cadherin, invasion, knock-down

Gliomas are the most common malignant tumors in the central nervous system and are characterized by highly aggressive growth [1]. The highly invasive nature of glioma contributes to the diffuse infiltration and migration of glioma cells. Thus, the glioma regularly results in restrictive and incomplete surgical resection in the surrounding glioma tissues. Although enormous improvements have been made in neurosurgery, chemotherapy, and radiotherapy, the prognosis of patients with glioma is still poor. [1-3]. Previously, more and more evidence demonstrated that epithelial-mesenchymal transition (EMT) was tightly involved in enhancing the invasive capability of gliomas and was correlated with histologic malignancy. During EMT, the cancer cells obtained plasticity with mesenchymal-type movement and became highly invasive. Hence, EMT may be one of the most infusive fields in oncobiology, especially in invasion and metastasis of glioma. [4-7]. Therefore, a better understanding of the EMT in gliomas is a key to research effective therapeutic strategy that abolishes the metastasis and invasion of glioma cells.

Numerous studies indicated that massive cellular and molecular processes may play an essential role in the signaling networks of cell migration $[8,9]$. Ste20-like kinase (SLK) is one of the members of the Ste20 family which is located in the nucleus, cytosol, microtubules and centrosomes. It has been implicated in a variety of biological processes, such as growth, proliferation, survival and cell migration [10-13]. Previous studies have shown that the activation of SLK was required to regulate cell migration [14-16]. Recently, our study also confirmed the function of SLK in the signaling networks of cell migration. Additionally, we have shown that SLK was recruited to the leading edge of migrating cells and co-localized with adhesion complex components such as the microtubules, Racl and Paxillin [12]. In human breast cancers, the expression of dominant-negative SLK could result in impaired motility and invasion [15]. Taken together, all available evidence suggests that SLK might be a new biological marker for the development and progression of human cancers. Nevertheless, whether SLK is involved in the modulation of invasiveness of glioma cells still remains to be investigated.

Currently, we revealed that the expression of SLK was markedly higher in glioma tissues and cell lines. Meanwhile, SLKexpressionlevel was upregulated in clinicalgliomasamples and had a negative correlation with E-cadherin. Knockdown of SLK decreased the invasive ability of glioma cells. 


\section{Patients and methods}

Patients and tissue samples. Fresh-frozen human glioma specimens were gained and in accordance with an institutional review board (IRB) protocol approved by the Partners Human Research Committee at Affiliated Hospital of Nantong University from 2005 to 2012. Normal brain specimens were donated by individuals who died in traffic accidents and had no pathologically detectable lesions. All tumor tissues came from newly diagnosed glioma patients without chemotherapy or radiation therapy before sample collection. All tissues were immediately frozen in liquid nitrogen after surgery and stored at $-80^{\circ} \mathrm{C}$ until use.

Immunohistochemistry. The tissue sections were dehydrated with gradient ethanol and xylene dewaxing and soaked in $0.3 \%$ hydrogen peroxide for 10 minutes to block endogenous peroxidase activity. We treated the sections with $0.1 \mathrm{M}$ citrate buffer ( $\mathrm{pH} 6.0$ ) and heated them at $105^{\circ} \mathrm{C}$ in autoclave for 10 minutes to retrieve the antigen. After rinsing in PBS ( $\mathrm{pH} 7.2$ ), the sections were incubated with rabbit anti-human SLK antibody and anti-E-cadherin antibody for $2 \mathrm{~h}$ at room temperature. Then, the peroxidase reaction was visualized by incubating the sections with DAB $(0.02 \%$ diaminobenzidine tetrahydrochloride, $0.1 \%$ phosphate buffer solution, and $3 \% \mathrm{H}_{2} \mathrm{O}_{2}$ ) after washing in PBS. At last, sections were counterstained with hematoxylin, dehydrated, and mounted in resin mount. Immunohistochemical staining was done by two independent scientists and the staining was observed under a microscope. The percentage of glioma cells was scored as follows: $0,<1 \% ; 1,1-25 \% ; 2$, 26-50\%; 3, 51-75\%; and 4, >75\%. The staining intensities were as follows: 1 (weak staining), 2 (moderate staining), and 3 (strong staining). From each of the two scale scores, each section was classified as: low/no SLK/E-cadherin expression or high SLK/E-cadherin expression.

Western blot analysis. Glioma tissues and cell proteins were homogenized instantly in a homogenization buffer containing $1 \mathrm{M}$ Tris $\mathrm{HCl}$ pH 7.5, 1\% Nonidet p-40 (NP-40), $1 \%$ Triton X-100, $10 \%$ sodium dodecyl sulfate (SDS), 0.5 M EDTA, 0.5\% sodium deoxycholate, leupeptin $10 \mu \mathrm{g} / \mathrm{mL}$, aprotinin $10 \mu \mathrm{g} / \mathrm{mL}$, and $1 \mathrm{mM}$ PMSF, and then centrifuged for 30 minutes to gather the supernatant liquid. The supernatant was deliquated in $2 \times \mathrm{SDS}$ loading buffer and denatured at $100^{\circ} \mathrm{C}$ for 15 minutes. An equivalent number of proteins from each specimen was electrophoresed by $10 \%$ sodium dodecyl sulfate-polyacrylamide gel electrophoresis (SDS-PAGE), then transferred to the PVDF membrane (Millipore, Bedford, Tumor Biol.MA). Being blocked in 5\% non-fat milk after Tris Physiological Buffered Saline and Tween 20 (TBST) (20 mM Tris, $150 \mathrm{mM} \mathrm{NaCl}, 0.05 \%$ Tween-20) for $2 \mathrm{~h}$ at room temperature, the membranes were incubated overnight at $4^{\circ} \mathrm{C}$ with the primary antibodies. After washing with TBST for three times, each time for 5 minutes, the membranes were incubated with horseradish peroxidase-linked IgG as the secondary antibody for $2 \mathrm{~h}$ at room temperature. The membrane was developed using the ECL detection systems. The experiments were conducted on three different occasions.

Antibodies. The antibodies used in this study included the following: anti-SLK antibody (1:300; Santa Cruz Biotechnology), E-cadherin (1:500; Santa Cruz Biotechnology), glyceraldehyde-3-phosphate dehydrogenase (GAPDH) (1:1000; Santa Cruz Biotechnology), Vimentin (1:1000; Santa Cruz Biotechnology), Snail (1:1000; Santa Cruz Biotechnology).

Cell culture. Glioma cell lines, including U87, U251, H4, and U118 were purchased from Cell Library, China Academy of Science, and cultured in Dulbecco's modified Eagle's medium (DMEM) (Gibco BRL, Grand Island, NY) with $10 \%$ fetal bovine serum, $2 \mathrm{mM}$ L-glutamine and $100 \mathrm{U} / \mathrm{ml}$ penicillin-streptomycin mixture (GibCo BRL) at $37^{\circ} \mathrm{C}$ with $5 \% \mathrm{CO}_{2}$. The medium was replaced every $2-3$ days, and cultures were split using $0.25 \%$ trypsin.

Expression plasmid and transient transfection. All oligonucleotides containing the short hairpin RNA (shRNA) target sequences were designed and synthesized by Shanghai Genechem (China). The sequences are siSLK-1: 5'-GCCAUAACCAGAACCUGAA-3'; siSLK-2:5'-GGUUGAGAUUGA CAUAUUA-3'. Transient transfections were performed using Lipofectamine 2000, following the manufacturer's recommendation (Life Technologies).

Transwell migration assay. Glioma cells were transfected with SLK shRNAs and starved overnight in DMEM media with $0.1 \%$ FBS and then trypsinized and resuspended into DMEM containing $0.1 \%$ bovine serum albumin. Cells $\left(1 \times 10^{5}\right)$ were added to the apical chamber of 24-well transwell plates (Corning, $8-\mu \mathrm{m}$ pore size), and DMEM with 10\% FBS was added to the bottom chambers. After cultured overnight, top (non-migrated) cells were removed and bottom (migrated) cells were fixed. Then the cells were stained with crystal violet to visualize nuclei. The number of migrating cells of five fields was counted under $\times 200$ magnification, and the means for each chamber were identified. All experiments were carried out in triplicate and repeated twice.

Monolayer wound-healing assays. Glioma cells were seeded to almost complete confluence in a monolayer in 6-well plates and grown to confluence overnight. After transfected forty-eight hours, cells were serum starved for $12 \mathrm{~h}$ and then scratched within the confluent cell layer by using a $10-\mu \mathrm{L}$ micropipette tip. Then the cells were cultured in $5 \%$ fetal bovine serum-Dulbecco's modified eagle medium (FBS-DMEM) at $5 \% \mathrm{CO}_{2}$ and $37^{\circ} \mathrm{C}$. Collected pictures at 0 , 24 , and 48 h time points by an inverted Leica phase-contrast microscope (Leica DFC 300 FX).

Statistical analysis. All statistical analyses were performed with SPSS 13.0 software (SPSS, Inc, Chicago, IL). The Chi square $\left(\chi^{2}\right)$ test was used to analyze the relationship between SLK expression and E-cadherin expression and the clinical features expression. The expression of SLK and E-cadherin was studied by using the Spearman rank correlation test 
because the data were not in widespread distribution. The Kaplan-Meier survival plots were used to analyze survival data, and curves were compared using the log-rank test. Data were calculated as (mean $\pm \mathrm{SD}$ ). P-values $<0.05$ in all cases were considered to be statistically significant.

\section{Results}

SLK overexpression in primary glioma. To confirm whether SLK was expressed in gliomas, our study explored the expression of SLK in normal brain tissue specimens and fresh glioma tissues by western blot. The results revealed that the expression level of SLK, at both protein and mRNA levels, was markedly higher in glioma tissues compared with normal brain tissues and increased with the malignant degree (Figure 1A, B). To further analyze the association of SLK with clinicopathologic grades of gliomas, we examined 104 paraffin-embedded glioma tissues, including 38 cases of grade II gliomas, 36 cases of grade III gliomas, and 30 cases of grade IV gliomas by immunohistochemistry. As shown in Figure 1C, SLK staining intensity from grade II to grade IV tumors was significantly higher than in normal brain tissues and its expression increased as the malignant degree increased. These data show that the expression of SLK is significantly associated with glioma grades.

Correlation between the expression of SLK and glioma patients' survival. We further analyzed the connection between clinical relevance of SLK and glioma patients' survival by summarizing the clinicopathological data of patients (Table 1). The percentage of the SLK positive cells in each specimen was calculated in the 104 samples using immunohistochemical evaluation. As presented in Figure 2A, we found that the expression level of SLK was strongly related to the clinicopathologic grades $(\mathrm{p}=0.031)$. Further analysis showed that no significant correlation was observed between SLK expression and patient's age, gender, extent of resection or tumor size in 104 glioma cases. However, its expression was negatively correlated with E-cadherin expression, which

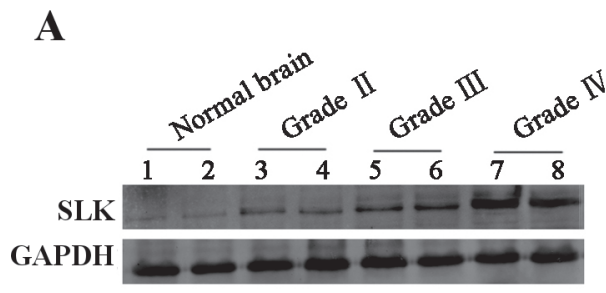

C
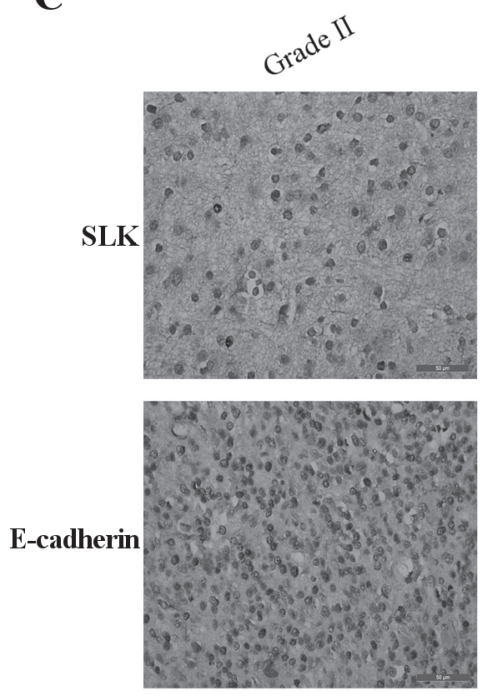

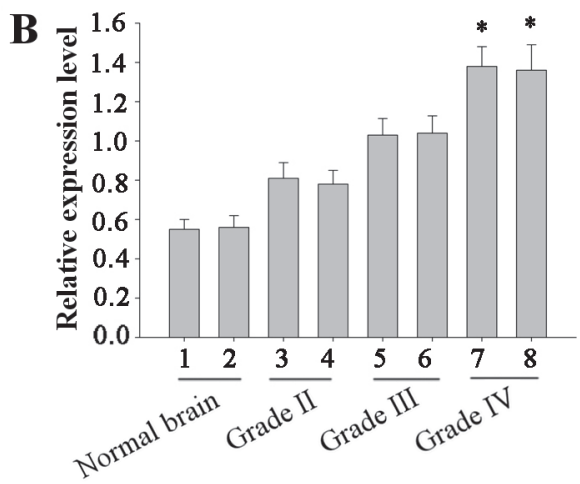

Grade
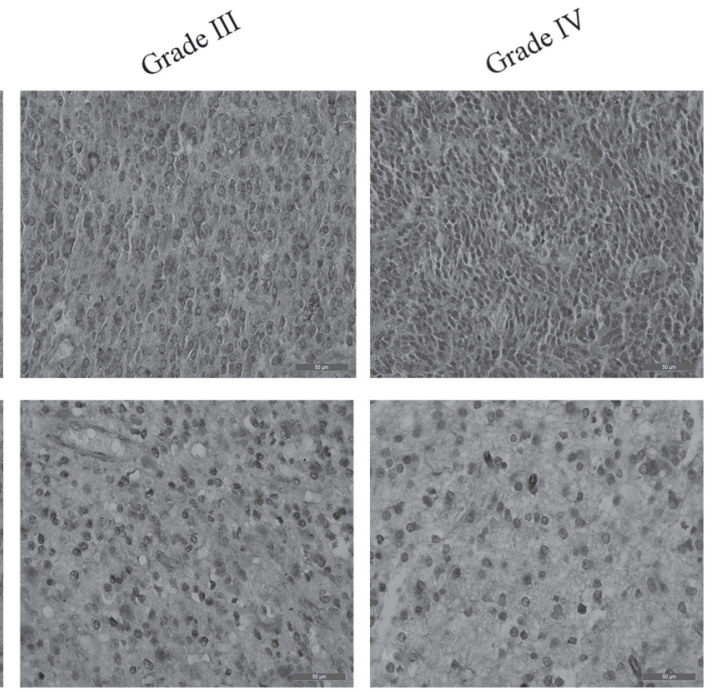

Figure 1. Expression of SLK in human glioma tissues. (A) Expression of SLK protein in two normal brain tissues and glioma tissues (grades II-IV). (B) The bar chart shows the ratio of SLK protein to GAPDH by densitometry. The data are mean \pm SD, $p<0.05$. (C) Paraffin-embedded glioma tissue sections (grades II-IV) were stained with SLK antibodies and anti-E-cadherin antibodies followed by counterstaining with hematoxylin (SP $\times 400)$. 
A

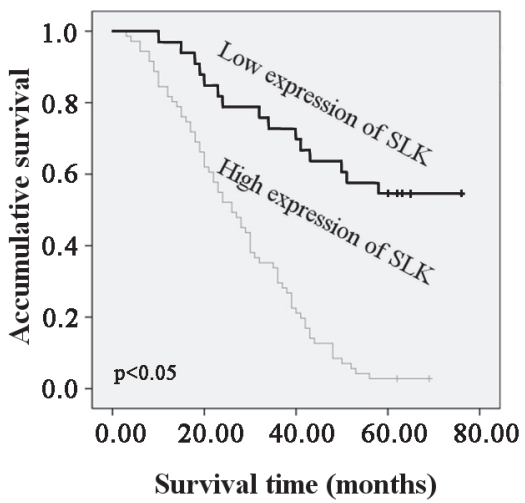

B

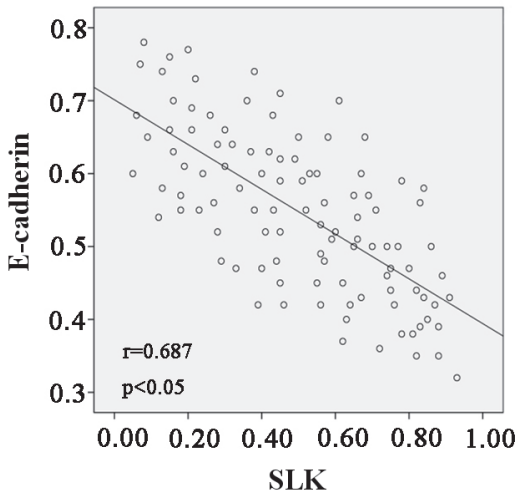

Figure 2. SLK correlated with the glioma patients' survival. (A) Kaplan-Meier postoperative survival curve for high SLK expression and low SLK expression in 104 patients of glioma. Patients in the high-expression of SLK group had poor survival. $(\mathrm{p}<0.05)$. (B) The relationship between SLK and E-cadherin.

Table 1. SLK expression and clinicopathologic characteristics of 104 glioma specimens.

\begin{tabular}{|c|c|c|c|c|}
\hline \multirow{2}{*}{ Variables } & \multirow{2}{*}{ Total } & \multicolumn{2}{|c|}{ SLK expression } & \multirow{2}{*}{ p-value ${ }^{*}$} \\
\hline & & Low & High & \\
\hline \multicolumn{5}{|l|}{ Age } \\
\hline$<45$ & 41 & 14 & 27 & 0.669 \\
\hline$>45$ & 63 & 19 & 44 & \\
\hline \multicolumn{5}{|l|}{ Gender } \\
\hline Female & 45 & 16 & 29 & 0.464 \\
\hline Male & 59 & 17 & 42 & \\
\hline \multicolumn{5}{|l|}{ Tumor location } \\
\hline Frontal & 27 & 9 & 18 & 0.902 \\
\hline Parietal & 15 & 5 & 10 & \\
\hline Occipital & 10 & 4 & 6 & \\
\hline Temporal & 35 & 9 & 26 & \\
\hline Unknown & 17 & 6 & 11 & \\
\hline \multicolumn{5}{|l|}{ Surgery } \\
\hline Partial resection & 41 & 12 & 23 & 0.031 \\
\hline Gross total resection & 63 & 15 & 48 & \\
\hline \multicolumn{5}{|l|}{ Tumor diameter } \\
\hline$<4 \mathrm{~cm}$ & 36 & 19 & 17 & 0.001 \\
\hline$>4 \mathrm{~cm}$ & 68 & 14 & 54 & \\
\hline \multicolumn{5}{|l|}{ Necrosis } \\
\hline Absence & 84 & 15 & 69 & 0.000 \\
\hline Presence & 20 & 18 & 2 & \\
\hline \multicolumn{5}{|l|}{ WHO grade } \\
\hline II & 38 & 18 & 20 & 0.031 \\
\hline III & 36 & 9 & 27 & \\
\hline IV & 30 & 6 & 24 & \\
\hline
\end{tabular}

Statistical analyses were performed by the Pearson $\chi^{2}$-test ${ }^{*} \mathrm{p}<0.05$ was considered significant

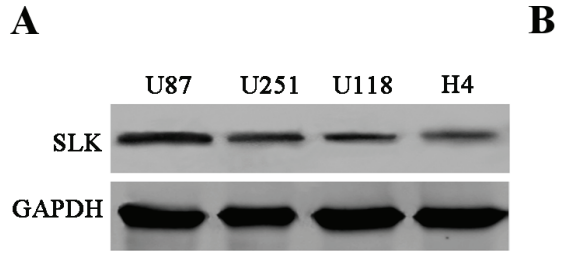

C

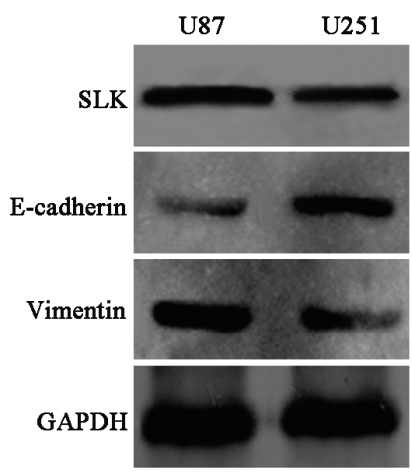

D

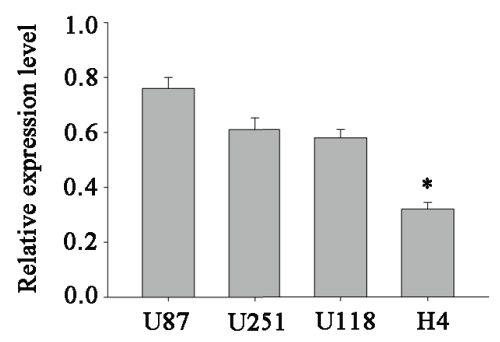

Con Vector Sh1 Sh2

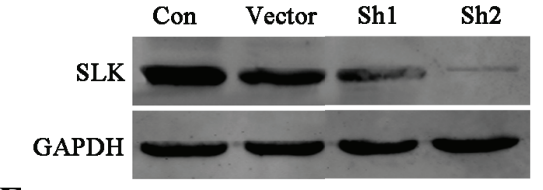

$\mathbf{E}$

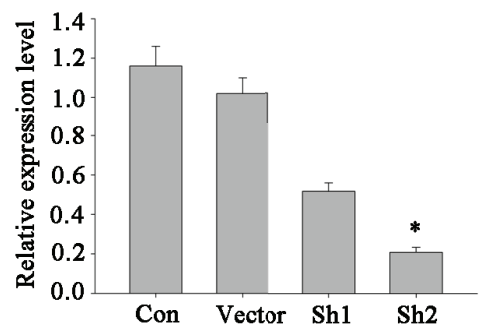

Figure 3. SLK regulated the EMT process of glioma cells. (A) Expression of SLK protein in cultured glioma cell lines (U87MG, U251MG, U118 and H4). (B) The bar chart demonstrates the ratio of SLK protein to GAPDH by densitometry. The data are mean \pm SD. (C) SLK, E-cadherin, Vimentin, and GAPDH protein in U87 and U251 cell lines analyzed by western blot analysis. (D) and (E) Silencing SLK expression by transfection with SLKsiRNA in U87 cells.

is the marker of epithelial cells (Figure 2B). The KaplanMeier survival curves revealed that high SLK level significantly correlated with poor survival.

Silencing SLK expression inhibited glioma cell migration. In order to explore the biological significance of endog- enous SLK in the development and progression of glioma cells, we examined the expression of SLK in human glioma cell lines by western blot analysis, including U87, U251, H4, and U118. As shown in Figure 3A and 3B, SLK was highly expressed in U87 and U251 cells. EMT is an important 
A

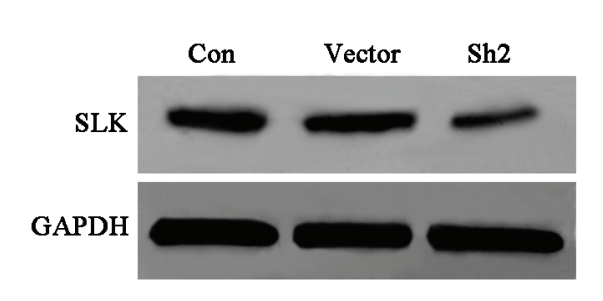

C

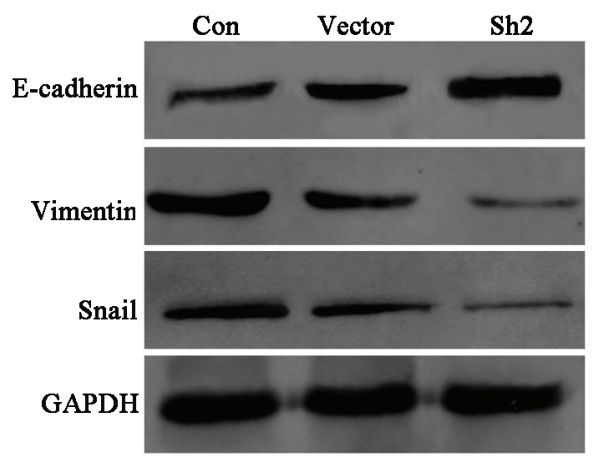

B

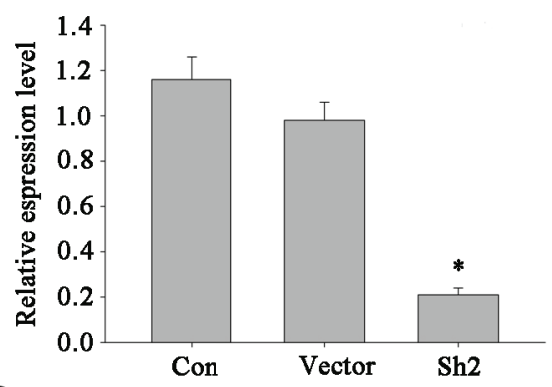

D

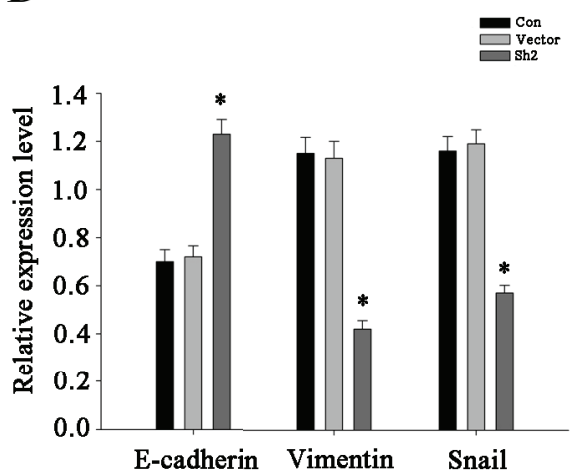

Figure 4. Silencing SLK expression downregulated E-cadherin expression in U87 cells. (A) and (B) Analysis of SLK in control, vector, sh2. (C) Western blot analysis of E-cadherin, Vimentin, and Snail in control, vector, and sh2 cells. (D) The bar chart shows the ratio of E-cadherin, Vimentin, and Snail to GAPDH by densitometry. The data are mean \pm SD. $p<0.05$.

process in glioma migration and invasion. Hence, we were prompted to examine the effects of SLK on the EMT process in human glioma cells. We studied the gene expression of two EMT marker proteins (E-cadherin, Vimentin) in glioma cells, and as presented in Figure 3C, the expression of E-cadherin was decreased in U87 cells. In contrast, the expression level of mesenchymal markers such as Vimentin was opposite to E-cadherin. Then, the dysfunction of SLK in human glioma cell models with RNA interference (RNAi)-mediated SLK knockdown was tested. U87 cells were transiently transfected with SLK-siRNA or control siRNA. $48 \mathrm{~h}$ later, siRNA targeting SLK markedly inhibited the expression of SLK protein in siSLK-2-transfected U87MG cells compared to the cells treated with siSLK-1 and control- siRNA (Figure 3D and 3E).

Therefore, we decided to use siSLK-2 to carry out the following experiments. As shown in Figure 4A and 4B, SLK expression was decreased in U87 cells transfected with siSLK-2. Then we found that knockdown of SLK increased E-cadherin expression and reduced Vimentin expression. Previous studies demonstrated that E-cadherin could be inhibited by Snail. Hence, we sought to explore the expression level of Snail. As expected, its expression was decreased in shSLK/U87MG cells (Figure 4C and 4D). These findings suggest that SLK regulats the EMT process that might contribute to glioma metastasis and invasion.

Modulation of the invasive ability of glioma cells by SLK in vitro. To determine whether the invasiveness of glioma cells was biologically and clinically related to the expression of SLK, representative photo-micrographs were taken at 0 , 24 , and 48 hours after the glioma cell surfaces were scratched. We found that silencing the endogenous SLK expression dramatically inhibited cell migration in glioma cell lines compared with the control and vector cells, as revealed by wound healing assay (Figure 5A and 5B). Furthermore, the SLK RNAi-transduced U87MG glioma cells markedly reduced the ability of glioma invasion compared with the vector control-transduced cells, as examined by transwell assays (Figure 5C and 5D). Taken together, these results provide evidence that SLK expression modulates the aggressiveness of glioma cells.

\section{Discussion}

The malignant glioma is usually associated with high morbidity and mortality and is a serious public health problem all over the world. The outstanding feature of aggressive growth of human gliomas has imposed some obstacles to thorough surgical removal of the tumor and might be the key factor leading to poor prognosis $[2,17-18]$. Currently, the study findings provided new insights into the potential role of SLK in promoting the invasiveness of gliomas.

Previous findings, including those made by ourselves, suggested that SLK was required for focal adhesion turnover and cell migration. SLK was required for invasion of breast 
$\mathbf{A}$

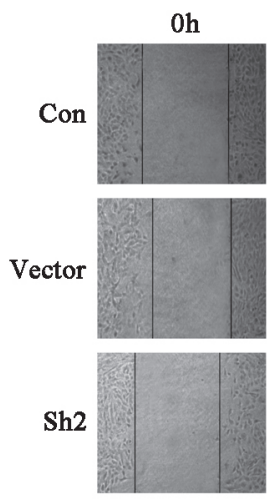

oh

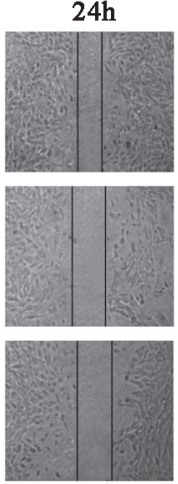

$4 h$

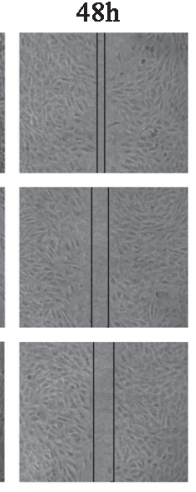

C

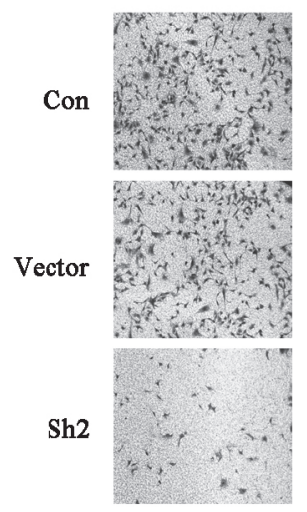

B

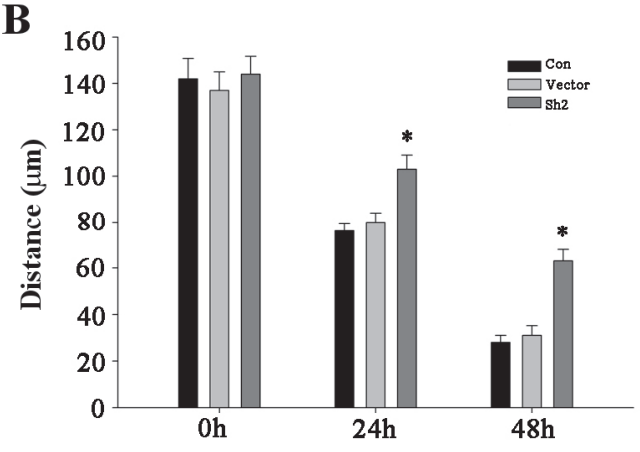

D

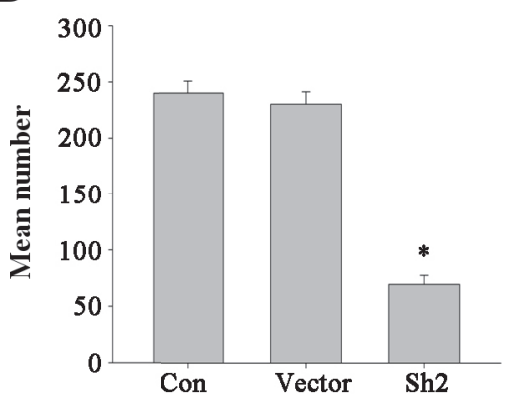

Figure 5. SLK modulated the invasive ability of glioma cells in vitro. (A) and (B) Wound healing assays with control, vector and transfected with the SLK siRNA cells. Migration of glioma cells to the wound was visualized at 0,24 , and $48 \mathrm{~h}$ with an inverted Leica phase contrast microscope $(\times 200 \mathrm{magnifica}$ tion). $\mathbf{p}<\mathbf{0 . 0 5}$. (C) and (D) Knockdown of SLK inhibited the migration ability in transwell assays. The quantification of penetrated cells was represented as the mean of three different experiments.

cancer cells, contributing to promotion of breast cancer progression and activation of relevant signaling pathways $[14,15]$. It promoted us to explore whether SLK plays a role in glioma invasion and has a prognostic effect on glioma patients. For this purpose, experiments were done to examine the effect of SLK expression on the invasion of glioma, as western blot analysis showed that SLK was markedly overexpressed in glioma tissue specimens. Together with the immunohistochemical results based on 104 glioma samples, the SLK expression significantly correlated with the WHO histologic grading $(\mathrm{p}=0.031)$. This result strongly indicates that SLK is involved in the progression of glioma and might be a new prognostic indicator of glioma. E-cadherin is a transmembrane glycoprotein that mediates homotypic intercellular adhesion and establishes interaction with intracellular proteins and coordination of epithelial cell morphology, polarity and function [19-21]. Knockdown of E-cadherin was related to metastasis and invasion of tumors including breast cancer and HCC [22]. Furthermore, previous study has reported that E-cadherin was down-regulated in the majority of glioma tissues. To access the relationship between SLK and EMT of glioma, the expression of E-cadherin in human glioma tissues was also evaluated. As shown in Figure 3 and 4, clearly positive expression of Vimentin and relatively weak expression of E-cadherin were detected in glioma cells. Especially, the SLK expression was found to be positively associated with the expression of Vimentin and negatively associated with E-cadherin expression, which suggested that SLK promoted the EMT of glioma.

To further validate the histological results, we altered the SLK expression in glioma cells by siRNA techniques to access its functional levels at the cell level. Interestingly, we found that silencing SLK drastically repressed E-cadherin expression accompanied by a down-regulation of Vimentin on mRNA and protein level in glioma cells. These results suggest that SLK could enhance the expression of Vimentin and repress the expression of E-cadherin in glioma cells. Epithelial-mesenchymal transition leading to malignant cancer progression has been confirmed to be correlated with loss of the epithelial traits and acquisition of the mesenchymal traits, such as increased expression of Vimentin and decreased expression of E-cadherin. Cancer cells with mesenchymal transition transformed into active cancer cells which increased invasive ability $[23,24]$. Both, transwell migration assay and monolayer wound-healing assays, showed that depletion of endogenous SLK drastically inhibited the glioma invasion. Hence, these results suggest that SLK-induced EMT in glioma cells promotes the invasiveness of glioma cells. 
In summary, our current study provides the first demonstration that SLK, a potential oncogene, is evidently overexpressed in gliomas and is associated with glioma grades as well as poor prognosis. Moreover, we found that silencing SLK expression reduced the ability of glioma invasion with a possible involvement of E-cadherin and Vimentin. Our findings indicate that SLK may be a novel therapeutic target for glioma. However, further study is required to explore the precise mechanism of SLK in the development of gliomas.

Acknowledgments: This work was supported by the National Natural Science Foundation of China (81572491).

\section{References}

[1] BUTOWSKI NA, SNEED PK, CHANG SM. Diagnosis and treatment of recurrent high-grade astrocytoma. J Clin Oncol 2006; 24: 1273-1280.

[2] SEE SJ, GILBERT MR. Anaplastic gliomas: diagnosis, prognosis, and management. J Clin Oncol 2004; 31: 618-634.

[3] WANG H, XU T, JIANG Y, XU H, YAN Y et al. The challenges and the promise of molecular targeted therapy in malignant gliomas. Neoplasia 2015; 17: 239-255. doi: 10.1016/j. neo.2015.02.002

[4] LAN L, LUO Y, CUI D, SHI BY, DENG W et al. Epithelialmesenchymal transition triggers cancer stem cell generation in human thyroid cancer cells. Int J Oncol 2013; 43: 113-120. doi: 10.3892/ijo.2013.1913

[5] LEE MY, CHOU CY, TANG MJ, SHEN MR. Epithelialmesenchymal transition in cervical cancer: correlation with tumor progression, epidermal growth factor receptor overexpression, and snail up-regulation. Clin Cancer Res 2008; 14: 4743-4750. doi: 10.1158/1078-0432.CCR-08-0234

[6] SONG E, YU W, XIONG X, KUANG X, AI Y et al. Astrocyte elevated gene-1 promotes progression of cervical squamous cell carcinoma by inducing epithelial-mesenchymal transition via Wnt signaling. Int J Gynecol Cancer 2015; 25: 345355. doi: 10.1097/IGC.0000000000000381

[7] ZOU M, ZHU W, WANG L, SHI L, GAO R et al. AEG-1/ MTDH-activated autophagy enhances human malignant glioma susceptibility to TGF- $\beta 1$-triggered epithelial- mesenchymal transition. Oncotarget 2016; 7: 13122-13138. doi: 10.18632/oncotarget.7536

[8] HEASMAN SJ, RIDLEY AJ. Mammalian Rho GTPases: new insights into their functions from in vivo studies. Nat Rev Mol Cell Biol 2008; 9: 690-701. doi: 10.1038/nrm2476

[9] RIDLEY AJ, SCHWARTZ MA, BURRIDGE K, FIRTEL RA, GINSBERG $\mathrm{MH}$ et al. Cell migration: integrating signals from front to back. Science 2003; 302: 1704-1709.

[10] DAVIS RJ. The mitogen-activated protein kinase signal transduction pathway. J Biol Chem 1993; 268: 14553-14556.
[11] FANGER GR, GERWINS P, WIDMANN C, JARPE MB, JOHNSON GL. MEKKs, GCKs, MLKs, PAKs, TAKs, and tpls: upstream regulators of the c-Jun aminoterminal kinases? Curr Opin Genet Dev 1997; 7: 67-74.

[12] WAGNER S, FLOOD TA, O'REILLY P, HUME K, SABOURIN LA. Association of the Ste20-like kinase (SLK) with the microtubule. Role in Rac1-mediated regulation of actin dynamics during cell adhesion and spreading. J Biol Chem 2002; 277: 37685-37692.

[13] ZINOVKINA LA, POLTARAUS AB, SOLOVYANOVA OB, NADEZHDINA ES. Chinese hamster protein homologous to human putative protein kinase KIAA0204 is associated with nuclei, microtubules, and centrosomes in CHO-K1 cells. FEBS Lett 1997; 414: 135-139.

[14] WAGNER S, STORBECK CJ, ROOVERS K, CHAAR ZY, KOLODZIEJ P et al. FAK/src-familydependent activation of the Ste20-like kinase SLK is required for microtubule-dependent focal adhesion turnover and cell migration. PLoS One 2008; 3: e1868. doi: 10.1371/journal.pone.0001868

[15] ROOVERS K, WAGNER S, STORBECK CJ, O'REILLY P, LO V et al. The Ste20-like kinase SLK is required for ErbB2driven breast cancer cell motility. Oncogene 2009; 28: 28392848. doi: 10.1038/onc.2009.146

[16] QUIZI JL, BARON K, AL-ZAHRANI KN, O'REILLY P, SRIRAM RK et al. SLK-mediated phosphorylation of paxillin is required for focal adhesion turnover and cell migration. Oncogene 2012; 32: 4656-4663. doi: 10.1038/onc.2012.488

[17] BUTOWSKI NA, SNEED PK, CHANG SM. Diagnosis and treatment of recurrent high-grade astrocytoma. J Clin Oncol 2006; 24: 1273-1280.

[18] LOUIS DN, POMEROY SL, CAIRNCROSS JG. Focus on central nervous system neoplasia. Cancer Cell 2002; 1: 125128.

[19] VAN ROY F, BERX G. The cell-cell adhesion molecule E-cadherin. Cell Mol Life Sci 2008; 23: 3756-3788. doi: 10.1007/s00018-008-8281-1

[20] BAUM B, GEORGIOU M. Dynamics of adherens junctions in epithelial establishment, maintenance, and remodeling. J Cell Biol 2011; 6: 907-917. doi: 10.1083/jcb.201009141

[21] CAPALDO CT, MACARA IG. Depletion of E-cadherin disrupts establishment but not maintenance of cell junctions in Madin-Darby canine kidney epithelial cells. Mol Biol Cell 2007; 18: 189-200.

[22] WANG Y, YANG S, NI Q, HE S, ZHAO Y et al. Overexpression of forkhead box J2 can decrease the migration of breast cancer cells. J Cell Biochem 2012; 113: 2729-2737. doi: $10.1002 / j \mathrm{cb} .24146$

[23] YANG J, WENBERG RA. Epithelial-mesenchymal transition: at the crossroads of development and tumor metastasis. Dev Cell 2008; 14: 818-829. doi: 10.1016/j.devcel.2008.05.009

[24] THIERY JP, ACLOQUE H, HUANG RY, NIETO MA. Epithelial-mesenchymal transitions in development and disease. Cell 2009; 139: 871-890. doi: 10.1016/j.cell.2009.11.007 